Check for updates

Cite this: RSC Adv., 2017, 7, 27913

\title{
Interaction of human serum albumin with uremic toxins: a thermodynamic study $\dagger$
}

\author{
Shun Yu, (D) abc Mirjam Schuchardt, ${ }^{d}$ Markus Tölle, ${ }^{d}$ Markus van der Giet, ${ }^{d}$
} Walter Zidek, ${ }^{d}$ Joachim Dzubiellaabc and Matthias Ballauff*abc

\begin{abstract}
We present a comprehensive study of the interaction of human serum albumin (HSA) with two uremic toxins, namely phenylacetic acid (PhAA) and indoxyl sulfate (IDS) in aqueous solution. The interaction of HSA with PhAA is studied for a series of salt concentrations (20-150 mM) and temperature $(25,30$ and $37^{\circ} \mathrm{C}$ ). The effect of in vitro urea modification of HSA upon its binding affinity towards the uremic toxins, is studied under the highest and lowest salt concentrations and the different temperatures. Isothermal titration calorimetry (ITC) is used to study the interaction by analyzing binding affinities and related thermodynamic data. It is found that two PhAA molecules bind to HSA in a sequential binding process with a binding constant $k_{\mathrm{b}}$ in the order of $\approx 10^{4}$ and $\approx 10^{3}$ for the first and second binding respectively. In contrast, IDS binds much stronger to HSA with a total of $\approx 3$ molecules to a high and low affinity binding site in the order of $\approx 10^{5}$ and $\approx 10^{3}$. Binding of uremic toxins to HSA in all cases show a decreasing binding affinity trend with increasing temperature and higher ionic strength. Thus binding of a second uremic toxin is strongly weakened at $37^{\circ} \mathrm{C}$ and $150 \mathrm{mM}$. Urea induced HSA modification have only minor effect on the binding interaction of the uremic toxins.
\end{abstract}

Received 8th March 2017 Accepted 19th May 2017

DOI: $10.1039 / \mathrm{c} 7 \mathrm{ra02838e}$

rsc.li/rsc-advances interaction to HSA are $p$-cresyl sulfate (PCS), hippuric acid (HA) and indoxyl sulfate (IDS)..$^{7,8}$ Bound to HSA, these toxins will inhibit the transport ability of the protein and may lead to structural modifications. ${ }^{4,5,9}$ Most toxins are found to interact with either one or both of the hydrophobic binding sites Sudlow I and II of HSA located in the subdomains IIA and IIIA. ${ }^{\mathbf{1 0 - 1 3}}$ Due to their tight binding, these small protein-bound and hydrophobic molecules are poorly cleared during conventional dialysis. ${ }^{\mathbf{1 4}, 15}$ The insufficiency of conventional dialysis in the removal of protein-bound toxins presents therefore a major challenge and improving renal dialysis by solving this problem is thus a central task of clinical nephrology. ${ }^{15-17}$ For this purpose an improved thermodynamic understanding of the interaction of toxins with HSA is absolutely necessary.

The obvious clinical importance of toxins has led to a number of studies of their interaction with proteins. Isothermal titration calorimetry (ITC) is the method of choice to measure enthalpy, entropy and binding affinity directly. ${ }^{18-21}$ Thus, calorimetric and spectroscopic methods have proven to be very suitable to characterized binding thermodynamics of ligands to proteins, namely ITC $^{\mathbf{1 0 , 1 2 , 1 3 , 2 2 - 2 5}}$ and fluorescence spectroscopy. ${ }^{\mathbf{1 0 , 1 2 , 1 3 , 2 3 - 2 5}}$ Other groups characterized binding isotherms by equilibrium dialysis (ED), ${ }^{\mathbf{3 , 4 , 1 3 , 2 6 , 2 7}}$ capillary electrophoresis $(\mathrm{CE})^{28-30}$ and chromatographic techniques. ${ }^{6,27,31} \mathrm{Up}$ to now, however, only a few studies have explored the dependence on temperature..$^{11,13,22,24}$ Much less is known about the dependence of the binding strength on ionic strength ${ }^{\mathbf{2 4 , 3 2}}$ in detail. However, Böhringer et al. have recently demonstrated in

\footnotetext{
${ }^{a}$ Soft Matter and Functional Materials, Helmholtz-Zentrum Berlin, Hahn-Meitner Platz 1, 14109 Berlin, Germany. E-mail: matthias.ballauff@helmholtz-berlin.de ${ }^{b}$ Helmholtz Virtual Institute "Multifunctional Biomaterials for Medicine", Kantstr. 55, 14513 Teltow, Germany. Tel: +49308062 43071

'Institut für Physik, Humboldt-Universität zu Berlin, Newtonstr. 15, 12489 Berlin, Germany

${ }^{d}$ Medizinische Klinik für Nephrologie, Universitätsmedizin Berlin, Campus Benjamin Franklin, Hindenburgdamm 30, 12203 Berlin, Germany

$\dagger$ Electronic supplementary information (ESI) available. See DOI: 10.1039/c7ra02838e
} 
an in vitro study that the fraction of uremic toxins was significantly decreased through hypertonic predilution hemodiafiltration. ${ }^{16}$ This finding underscores clearly the importance of ionic strength for the binding of toxins to HSA.

In a previous investigation we have explored both the effect of temperature and ionic strength on the binding of a polymeric toxin to HSA. ${ }^{33}$ There we studied the interaction between an oligmeric poly(acrylic acid) (PAA) and HSA using ITC. PAA provides a model molecule for a charged middle molecular weight toxin bearing carboxylic groups. We found a strong dependence of the binding strength (near the Sudlow II site) on ionic strength which clearly revealed the importance of ionic contributions to polyelectrolyte binding to protein.

In the present study we present a comprehensive study of the binding of small hydrophobic uremic toxins to HSA, again using ITC. Phenylacetic acid (PhAA) is a toxin that has not yet attracted much attention so far. The importance of this toxin derives from the fact that quantitative experiments using equilibrium dialysis (ED) and chromatographic methods have revealed a considerable amount of PhAA present in dialysis patients prior and after dialysis. ${ }^{6,14,16}$ Furthermore, in a high performance liquid chromatography experiment (HPLC), it has been shown that PhAA accumulation in CKD patients inhibits the expression of inducible nitric oxide synthase and therefore might contribute to the higher cardiovascular risk of these patients. $^{2}$ The importance of ionic contributions to the binding of this toxin is demonstrated in an in vitro study by Böhringer et al. and forms the basis for establishing a more effective clearance of PhAA by raising the ionic strength. ${ }^{16}$ Certainly, the binding behavior in an in vivo environment of blood plasma is more a complex process where the specific binding mechanisms cannot be identified anymore.

Indoxyl sulfate is studied as a second toxin because it bears a strong acidic $\left(\mathrm{SO}_{4}{ }^{-}\right)$group and studies have found that that $94 \%$ of IDS is protein bound and the removal after dialysis is only around $50 \% .^{8,34}$ Hence, a comparison of the thermodynamic data of PhAA bearing a weak acidic group $\left(\mathrm{COO}^{-}\right)$with the ones obtained from IDS can reveal details of the ionic interaction and its relation to toxin binding.

It is furthermore known from literature that HSA is modified in pathophysiological conditions caused by chronic renal failure $^{35}$ Thus, urea-induced carbamylation of proteins, including HSA, on multiple lysine- and arginine-containing chains occurs. The concentration of urea is chronically elevated in patients with renal failure and the subsequent protein modification was identified as risk factor for mortality. ${ }^{35}$ The modification of serum albumin might affect binding affinities of uremic toxins and therefore impair its dialysis condition and its subsequent chronically elevated plasma concentrations. We therefore explored the effect of ureamodification on binding affinities of PhAA and IDS to HSA and their structure upon complex formation. The entire thermodynamic analysis based on a comprehensive investigation by ITC will provide a firm basis for further clinical studies related to an improved dialysis technique.

\section{Experimental}

\subsection{Materials}

Phenylacetic acid (PhAA) and indoxyl sulfate potassium salt (IDS) were purchased from Sigma-Aldrich (Schnelldorf, Germany) and used as received. Human serum albumin (HSA) was also purchased from Sigma-Aldrich (lyophilized powder, fatty acid free, globulin free, 99\%) with molecular weight calculated MW $=66400 \mathrm{~g} \mathrm{~mol}{ }^{-1}$ and its purity verified by SDSgelelectrophoresis. The buffer morpholin- $N$-oxide (MOPS) was purchased from Sigma-Aldrich and used as received.

\subsection{In vitro urea modification of albumin}

Albumin as purchased by Sigma-Aldrich was dissolved and dialysed against buffer prior to use. In vitro modification of HSA was done in a $10 \mathrm{mM}$ MOPS buffer solution with $15 \mathrm{~g} \mathrm{~L}^{-1} \mathrm{HSA}$ and $10 \mathrm{mM}$ urea at $37^{\circ} \mathrm{C}$ and $\mathrm{pH} 7.4$ for $18 \mathrm{~h}$. After incubation, the mixture was dialyzed against buffer using centrifugation dialysis several times.

2.2.1 Isothermal titration calorimetry. ITC experiments were performed using a VP-ITC instrument (Microcal, Northampton, MA). All samples were prepared in a pH 7.4 buffer solution using $10 \mathrm{mM}$ MOPS and $10 \mathrm{mM}$ or $140 \mathrm{mM} \mathrm{NaCl}$ to adjust ionic strength. The correct $\mathrm{pH}$ for PhAA solutions were adjusted by adding minor amount of $\mathrm{NaOH}$. All samples were degassed prior to experiment and protein solutions were dialyzed against a $10 \mathrm{mM}$ buffer solution with corresponding $\mathrm{pH}$ using the dialysis-system Float-a-Lyzer by Spectrum Labs with molecular weight cut-off (MWCO) $20 \mathrm{kDa}$. The concentration of the protein solution after dialysis was determined using the characteristic absorption at $280 \mathrm{~nm}$ by UV-vis spectroscopy. Thus concentration errors are within the range of $\pm 0.1 \mathrm{~g} \mathrm{~L}^{-1}$. The samples were thermostatted and the instrument stabilized for 30 minutes to ensure thermal equilibrium and stability of the system. A total of $276 \mu \mathrm{L}$ PhAA solution was titrated with 20 successive $3 \mu \mathrm{L}$ injections followed by 36 successive $6 \mu \mathrm{L}$ injections, to acquire accurate binding data for low molar ratios. Stirring was performed with $307 \mathrm{rpm}$ and a time interval of 300-350 s was set between each injection into the cell containing $1.4 \mathrm{~mL}$ protein solution. The concentration of PhAA, IDS and HSA were $0.8 \mathrm{~g} \mathrm{~L}^{-1}$, $0.5 \mathrm{~g} \mathrm{~L}^{-1}$ and $1 \mathrm{~g} \mathrm{~L}^{-1}$ respectively. The concentration of HSA was determined using UV. The experiments were performed at 25, 30, $37^{\circ} \mathrm{C}$ and ionic strengths of $20 \mathrm{mM}$ and $150 \mathrm{mM}$.

As a first step of the ITC data analysis, the integration of the measured heat $Q$ over time is carried out to obtain the incremental heat $\Delta Q$ as a function of the molar ratio $x$ between toxin and protein. For each experiment, the heat of dilution of PhAA and IDS were measured separately by titrating the equivalent uremic toxin into blank buffer solution and subtracted from adsorption heats. After correction, the resulting binding isotherm are fitted using a supplied module for Origin ${ }^{\circledR} 7.0$ (Microcal). A brief overview of the fitting models used will be given below, however, the interested reader is referred to the manual by Origin for the details of the fitting procedure. ${ }^{36}$

Errors in concentrations when preparing the samples are seen as a very common source of error directly affecting the 
analysis of the isotherm and thus all thermodynamic values. ${ }^{37-39}$ However, in the present study both protein and toxin concentration are well defined.

2.2.2 Analysis of ITC data. When the protein P has only one binding site to which a single ligand L binds, the Single Set of Identical Sites model (SSIS) is used, and the binding process can be expressed by: ${ }^{\mathbf{4 0 - 4 2}}$

$$
\mathrm{P}+\mathrm{L} \stackrel{K_{\mathrm{b}}}{\longleftrightarrow} \mathrm{PL} \quad K_{\mathrm{b}}=\frac{[\mathrm{PL}]}{[\mathrm{P}][\mathrm{L}]}
$$

where $K_{\mathrm{b}}$ is the binding constant and characterizes the binding process, $[\mathrm{P}]$ the concentration of free sites on the protein, $[\mathrm{L}]$ the free ligand concentration and $[\mathrm{PL}]$ the concentration of the complex. This model is related to the Langmuir adsorption model which assumes an equilibrium between the empty adsorption sites, the number of proteins in solution and the occupied adsorption sites. This leads to the definition of a saturation parameter $\Theta$, that denotes the fraction of sites occupied by the ligand and the concentration of free ligand [L] in solution. $\Theta$ is related to the binding affinity $K_{\mathrm{b}}$ as follows:

$$
\Theta=\frac{K_{\mathrm{b}}[\mathrm{L}]}{1+K_{\mathrm{b}}[\mathrm{L}]}
$$

Since only the total concentrations of ligand $[\mathrm{L}]_{\mathrm{t}}$ and protein $[\mathrm{P}]_{\mathrm{t}}$ in the solution is known, $[\mathrm{L}]$ is expressed as:

$$
[\mathrm{L}]_{\mathrm{t}}=[\mathrm{L}]+N \Theta[\mathrm{P}]_{\mathrm{t}}
$$

with $N$ as the number of sites per protein.

For a more complicated binding process, where the protein has two non-identical binding sites, we choose to use a stepwise binding model, which is referred to as Sequential Binding Sites (SBS) model. Here macroscopic binding constants $K_{\mathrm{b} 1}$ and $K_{\mathrm{b} 2}$ refer to a first equilibrium state where one ligand binds to any of the two sites with $K_{\mathrm{b} 1}$ followed by a second stage where the second ligand binds to the remaining free site with $K_{\mathrm{b} 2}$ :

$$
K_{\mathrm{b} 1}=\frac{[\mathrm{PL}]}{[\mathrm{P}][\mathrm{L}]} \quad K_{\mathrm{b} 2}=\frac{\left[\mathrm{PL}_{2}\right]}{[\mathrm{PL}][\mathrm{L}]}
$$

The interpretation of this model must be carried out with care, as misinterpretations of $K_{\mathrm{b} 1}$ and $K_{\mathrm{b} 2}$ being "high" and "low affinity" respectively, are found in literature. ${ }^{43}$

Binding processes that involve more than exactly one ligand to a corresponding site can be described by the Two Sets of Independent Sites (TSIS) model. Two independent binding constants with corresponding saturation parameters are defined as follows:

$$
k_{\mathrm{b} 1}=\frac{\Theta_{1}}{\left(1-\Theta_{1}\right)[\mathrm{L}]} \quad k_{\mathrm{b} 2}=\frac{\Theta_{2}}{\left(1-\Theta_{2}\right)[\mathrm{L}]}
$$

The total ligand concentration in eqn (3) then becomes:

$$
[\mathrm{L}]_{\mathrm{t}}=[\mathrm{P}]+[\mathrm{P}]_{\mathrm{t}}\left(n_{1} \Theta_{1}+n_{2} \Theta_{2}\right)
$$

In contrast to the SBS model above, in this case $k_{\mathrm{b} 1}$ and $k_{\mathrm{b} 2}$ are microscopic binding constants describing the binding affinity of a ligand to the corresponding site. In this model, six free parameters are involved. Namely for each site two enthalpies $\Delta H_{i}^{\mathrm{ITC}}$, the binding numbers $n_{i}$ to each site $i$ and binding constants $k_{\mathrm{b} i}$ respectively.

In an ITC experiment, the total heat of adsorption $q$ is measured typically as an differential heat $\mathrm{d} q$ divided by the moles of ligand in the $j$-th titrant injected $\mathrm{d} l_{j}$ with $\mathrm{d} l_{j} \approx V_{\mathrm{t}} \mathrm{d}[\mathrm{L}]_{\mathrm{t}}$. The incremental heat $Q$ can be expressed as a differential equation: ${ }^{44}$

$$
Q=\frac{\mathrm{d} q}{V_{\mathrm{t}} \mathrm{d}[\mathrm{L}]_{\mathrm{t}}}=\sum_{m=1}^{n} \Delta H_{m} \frac{\mathrm{d}\left[\mathrm{PL}_{m}\right]}{\mathrm{d}[\mathrm{L}]_{\mathrm{t}}}
$$

where $n$ is the number of binding sites, $V_{\mathrm{t}}$ the cell volume and $\Delta H_{m}$ the molar heat of the reaction.

The fitting of the experimental data is performed by calculating the incremental heat $Q^{\prime}$ released with each titration and a correction for the displaced volume $\mathrm{d} V_{i}$ after each inject ion $i:^{36}$

$$
Q_{i}^{\prime}=Q_{i}+\frac{\mathrm{d} V_{i}}{V_{t}}\left[\frac{Q_{i}+Q_{i-1}}{2}-Q_{i-1}\right]
$$

Manually set initial values are used to compare with the measured isotherm. The initial values are then improved by using the standard Marquardt method. These steps are repeated iteratively until a satisfactory fit is achieved. ${ }^{36}$

The free energy of binding can be calculated for the corresponding binding process using the binding constant:

$$
\Delta G_{\mathrm{b}}=-R T \ln K_{\mathrm{b}}
$$

Furthermore, the van't Hoff enthalpy can be derived from a series of experiments conducted at different temperature:

$$
-R \frac{\partial \ln K_{\mathrm{b}}}{\partial\left(\frac{1}{T}\right)}=\Delta H_{\mathrm{b}}
$$

where the binding enthalpy $\Delta H_{\mathrm{b}}$ can be obtained from the slope of the linear fit and $\Delta S_{\mathrm{b}}$ from the intercept of the integrated form:

$$
\ln K_{\mathrm{b}}=-\frac{\Delta H_{\mathrm{b}}}{R T}+\frac{\Delta S_{\mathrm{b}}}{R}
$$

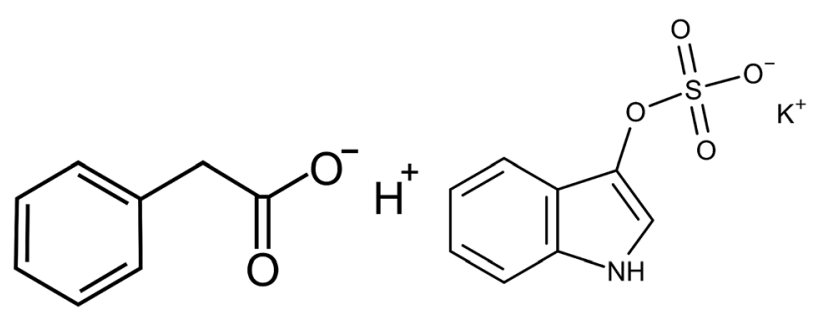

Fig. 1 Left: Phenylacetic acid (PhAA). Right: Indoxyl sulfate potassium salt (IDS) 
a)

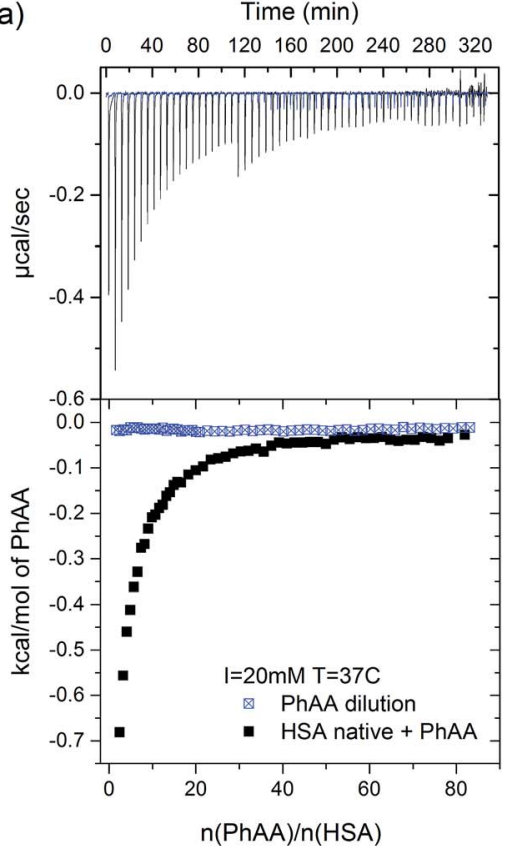

b)

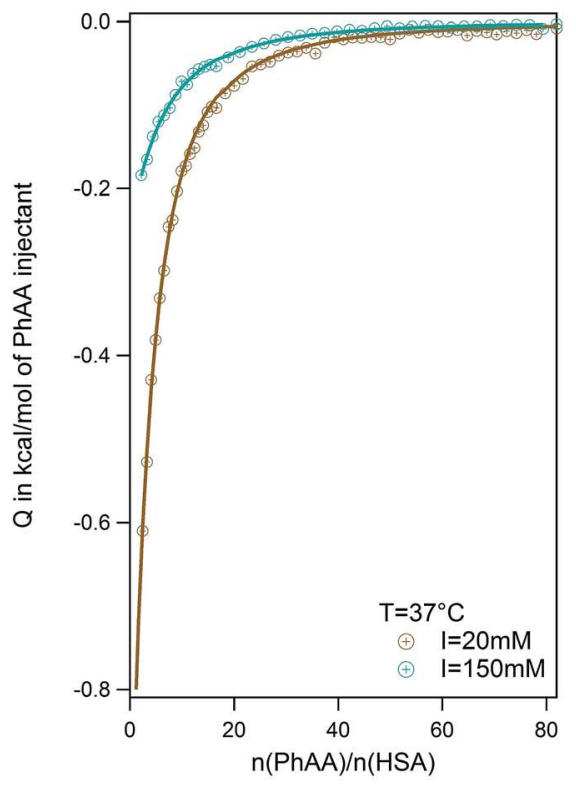

c)
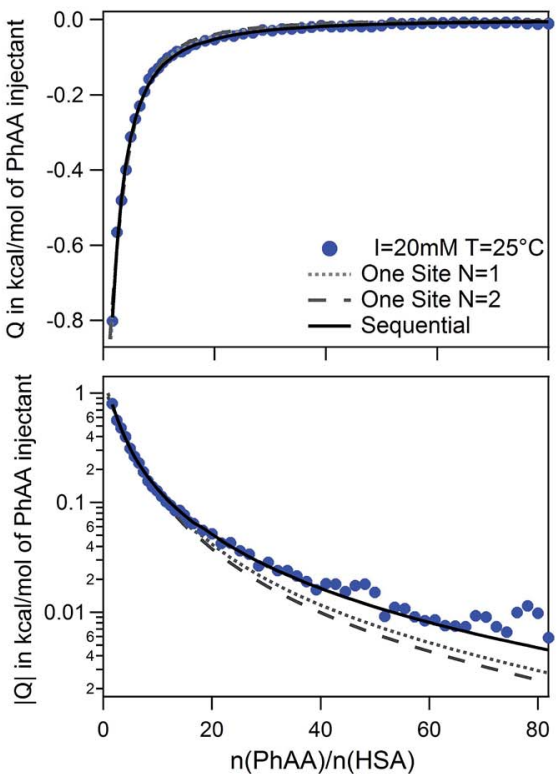

Fig. 2 PhAA adsorption to native HSA. (a) Calorimetric titration curves with differential heats per mole $Q$ are shown as a function of molar ratio $n(\mathrm{PhAA}) / n(\mathrm{HSA})$ for adsorption (black line and symbols) and the corresponding heats of dilution (blue line and symbols) at $I=20 \mathrm{mM}$ and $T=$ $37^{\circ} \mathrm{C}$. (b) Dilution corrected isotherms with corresponding fits are shown and the data for $150 \mathrm{mM}$ is compared to $20 \mathrm{mM}$ at $37^{\circ} \mathrm{C}$. (c) The quality of different fit models are demonstrated on the example of $20 \mathrm{mM}$ and $37^{\circ} \mathrm{C}$ data in a typical ITC plot (top) and a semi-logarithmic plot of $|Q|$ (bottom). Corresponding $\chi^{2}$ values for each fit is given in Table S1 of the ESI. $\dagger$

\section{Results and discussion}

\subsection{Binding of phenylacetic acid to native HSA}

We performed systematic series of ITC experiments with high (I $=150 \mathrm{mM})$ and low $(I=20 \mathrm{mM})$ ionic strengths and three different temperatures comprising room temperature $25{ }^{\circ} \mathrm{C}$, a intermediate temperature $30{ }^{\circ} \mathrm{C}$, and the physiological temperature $37{ }^{\circ} \mathrm{C}$. The experiments were performed at $\mathrm{pH} 7.4$ in buffer solution. Typical raw data with ITC titration peaks for adsorption (black curves and points) and dilution (blue curves and points) is shown exemplarily for the adsorption of PhAA to native HSA in Fig. 2a. The integrated isotherms were fitted after subtraction of the heat of dilution with either a Single Set of Independent Sites (SSIS) model, Sequential Binding Sites (SBS) model or Two Set of Independent Sites (TSIS) modelł in Fig. $2 \mathrm{~b}$.

All measured signals were weakly exothermic and exhibit similar monotonic curve progression as shown in Fig. $2 \mathrm{~b}$. This result is typically observed in the adsorption of small hydrophobic molecules to HSA in other studies as well. ${ }^{\mathbf{1 3 , 4 5 , 4 6}}$ Because the overall heat is rather small, dilution must be subtracted as a additional heat signal may affect the results of the fits.

3.1.1 Evaluation of ITC data. The evaluation of the ITC data is demonstrated on the example of PhAA adsorption to native HSA. In Fig. 2c we show the binding isotherm for adsorption at $25{ }^{\circ} \mathrm{C}$ and $20 \mathrm{mM}$ salt and corresponding fits with the two models described in the section ITC data analysis to evaluate

\$ All models were fitted using the implemented ITC data analysis software for Origin by MicroCal. the quality of the fits. Two fitting curves for fixed $N=1$ and $N=$ 2 (dotted and broken line respectively) with the SSIS model are compared to the SBS model for two sites. As the curves can hardly be discriminated in the common ITC diagram (as shown in the top of Fig. 2c), we plot the absolute value of ITC heats $|Q|$ on a logarithmic axis (bottom figure). At low molar ratio, all fitted curves coincide with the data. However, at higher PhAA concentrations, deviation of the different fits becomes more obvious and the importance of the high molar ratio data is evident. For the given case, data points are clearly best described by the SBS model, pointing towards the existence of two different binding sites of the HSA for PhAA. In the following we used this semi-logarithmic plot to determine the best fit available.

A few more examples are shown in Fig. S1 of the ESI. $\dagger$ Comparing the different binding isotherms and their fitting, it becomes evident that binding behavior is different for different temperature and ionic strength. At high temperature, both models converge towards each other (see Fig. S1b and $c^{\dagger} \dagger$ ). The affinity $K_{\mathrm{b} 2}$ to bind a second PhAA molecule to HSA becomes very weak for $20 \mathrm{mM}$ salt and $37{ }^{\circ} \mathrm{C}$ and vanishes for higher ionic strength at the same temperature (Fig. S1c and Tables S1, 2 ) in the ESI. $\dagger$ In this case, the data can be well described with the SSIS model with $N=1$. For other cases, where two binding sites are present, the SSIS model permanently underestimates binding affinity and enthalpy and produce a rather large error as seen in $\chi^{2}$. A comparison of the results of the fits is given in Table S1 of the ESI. $\dagger$ 
Table 1 Binding free energies $\Delta G_{\mathrm{b} i}$ of PhAA binding to native and modified HSA calculated according to eqn (9). $i$ denotes the first and second binding process in the SBS model

\begin{tabular}{|c|c|c|c|c|c|c|c|}
\hline & 50 & - & - & - & - & $-24.0 \pm 0.1$ & $-17.1 \pm 0.4$ \\
\hline & 70 & - & - & - & - & $-23.7 \pm 0.1$ & $-14 \pm 8$ \\
\hline & 100 & - & - & - & - & $-23.4 \pm 0.1$ & $-14.6 \pm 0.9$ \\
\hline & 150 & $-22.6 \pm 0.1$ & $-15.4 \pm 0.9$ & $-22.5 \pm 0.1$ & $-16.0 \pm 0.4$ & $-21.8 \pm 0.1$ & $-15.6 \pm 1.3$ \\
\hline
\end{tabular}

Table 2 Binding free energies $\Delta G_{\mathrm{b} i}$ of IDS binding to native and modified HSA, where $i$ denotes the two binding sites, respectively

\begin{tabular}{|c|c|c|c|c|c|c|c|}
\hline HSA state & $I, \mathrm{mM}$ & \multicolumn{2}{|l|}{$T=25^{\circ} \mathrm{C}$} & \multicolumn{2}{|l|}{$T=30^{\circ} \mathrm{C}$} & \multicolumn{2}{|l|}{$T=37^{\circ} \mathrm{C}$} \\
\hline & 150 & $-29.04 \pm 0.05$ & $-16.0 \pm 0.3$ & $-28.6 \pm 0.1$ & $-15.6 \pm 0.6$ & $-28.1 \pm 0.1$ & $-15.4 \pm 1$ \\
\hline Modified & $\begin{array}{l}20 \\
150\end{array}$ & $\begin{array}{l}-30.64 \pm 0.06 \\
-29.19 \pm 0.08\end{array}$ & $\begin{array}{l}-19.96 \pm 0.08 \\
-16.9 \pm 0.3\end{array}$ & $\begin{array}{l}-31.20 \pm 0.08 \\
-29.1 \pm 0.1\end{array}$ & $\begin{array}{l}-20.2 \pm 0.1 \\
-17.4 \pm 0.3\end{array}$ & $\begin{array}{l}-30.44 \pm 0.07 \\
-28.3+0.1\end{array}$ & $\begin{array}{l}-20.3 \pm 0.1 \\
-16 \pm 1\end{array}$ \\
\hline
\end{tabular}

3.1.2 Influence of temperature on the strength of binding. The dependence of the adsorption of PhAA onto HSA on ionic strength and temperature were studied to conduct a full thermodynamic analysis. Measurements at different temperatures are depicted exemplarily in Fig. 3 for $I=20 \mathrm{mM}$ ionic strength (isotherms for $I=150 \mathrm{mM}$ are shown in ESI Fig. S2 $\dagger$ ). The best fits determined as explained before are depicted as lines. In contrast to the adsorption of polyelectrolytes (PE) to HSA that has been investigated in earlier studies, ${ }^{33}$ the present binding curves do not exhibit a strong temperature dependence for

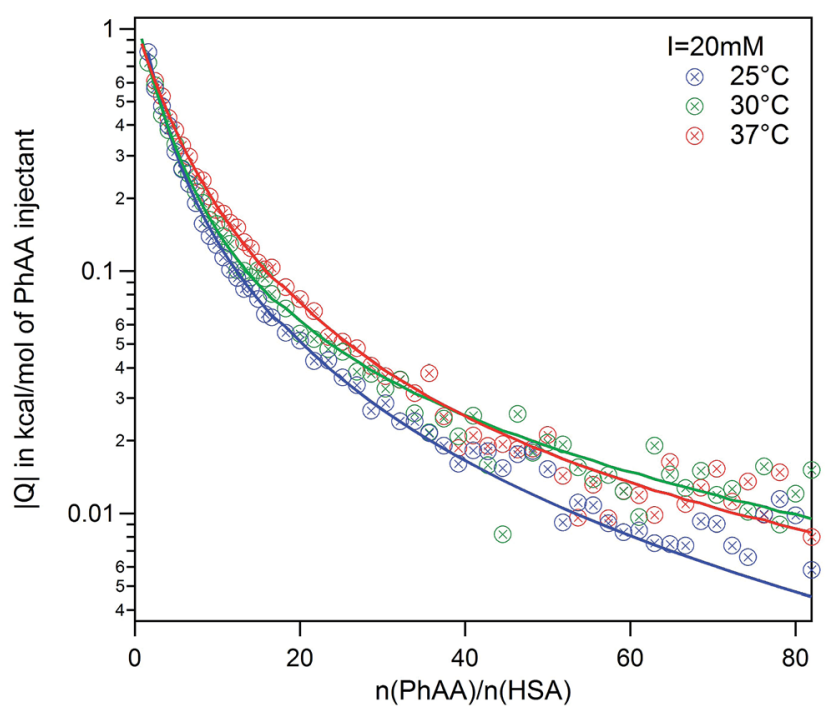

Fig. 3 PhAA adsorption to native HSA. Absolute heats $|Q|$ are shown for ionic strength $I=20 \mathrm{mM}$ at different temperatures and respective fits are displayed. either $20 \mathrm{mM}$ or $150 \mathrm{mM}$ ionic strength. Nevertheless, a consistent change in the curvature of the isotherms is obvious and also reflected in the binding parameters listed in Tables 1 and $\mathrm{S} 2$ of the ESI. $\dagger$

In general, the binding affinity decreases with increasing temperature as has been observed in several cases of adsorption of small molecules to HSA.,.$^{3,13,24,27}$ For example, Zaidi et al. have

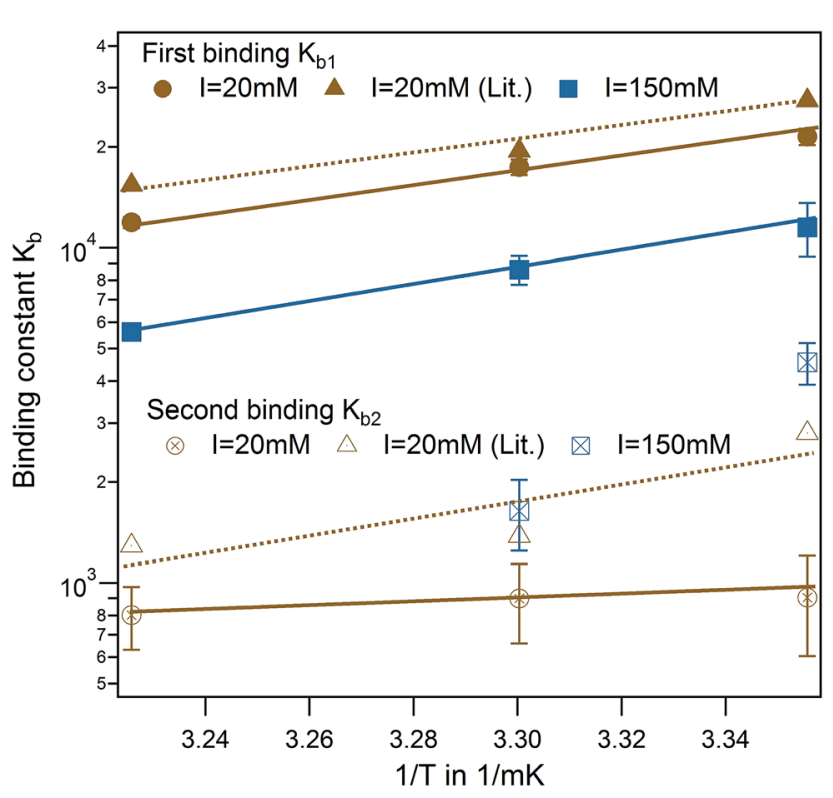

Fig. 4 Effect of temperature for PhAA binding to native HSA. Binding affinities for first (full symbols) and second binding process (open symbols) are shown in a van't Hoff plot for $I=20 \mathrm{mM}$ (brown) and $I=$ $150 \mathrm{mM}$ (blue) with corresponding fits according to eqn (10). Literature data for HA-HSA binding with $20 \mathrm{mM}$ sodium phosphate buffer $(\mathrm{pH}$ 7.4) are included as triangles with dotted lines as guideline for the eye. ${ }^{13}$ 
studied interaction between hippuric acid (HA) and HSA and their dependence on temperature using steady state fluorescence quenching measurements and ITC. The ITC data was analyzed using the SBS model likewise and reveal common trends for the temperature dependence of binding constant.

In a direct comparison of the literature data with the present results, binding affinity of the first binding site very much coincides in trend and magnitude as shown in Fig. 4 (brown triangle and spheres). ${ }^{13}$ For the second binding site, a minor dependence on temperature was observed in the case of PhAA adsorption to HSA. The dependence on temperature can be analyzed according to van't Hoff's law using eqn (10). This equation applies to a system where the change of heat capacity of the system $\Delta c_{\mathrm{p}}$ is small (see Table S2 of the ESI $\dagger$ ).

van't Hoff plots of the first and second ligand binding process are displayed in Fig. 4 and the results summarized in Table S2 of the ESI. $\dagger$ The analysis of the van't Hoff enthalpy done here is only semi-quantitative since a fully quantitative determination would require measurements at far more temperatures than done here. For the toxin IDS, the enthalpy as measured directly by ITC and van't Hoff enthalpy coincide quite well and $\Delta H_{\text {ITC }}$ is in the present study a good measure for the enthalpy of the binding. In the following, we focus on analysis of the binding free energy $\Delta G_{\mathrm{b}}$ calculated using eqn (9).

Another interesting feature is observed for $I=150 \mathrm{mM}$, where a decrease of a second ligand binding with temperature is measured. At high ionic strength and temperature both binding enthalpy and binding constant almost vanishes pointing towards an increased Coulombic screening and thus a weakening of the interaction. This is a plausible explanation for the results obtained recently by Jankowski et al. who measured almost a doubling of released PhAA during hypertonic predilution hemodiafiltration. ${ }^{16}$ Furthermore, we have shown in previous studies, that ionic strength does contribute significantly towards charge-charge interaction between protein and a short polyelectrolyte. ${ }^{33}$

3.1.3 Influence of ionic strength on binding. To gain more detailed insight into the effect of ionic strength, we performed three additional measurements at $37^{\circ} \mathrm{C}$ and $I=50 \mathrm{mM}, 70 \mathrm{mM}$ and $100 \mathrm{mM}$ (with ITC parameters listed in Table 1). We present exemplary isotherms in Fig. 5, while in the inset we plot the binding constant $K_{\mathrm{b} 1}$ against ionic strength. We find that the binding energy $\Delta G_{\mathrm{b} 1}$ is lowered only by $1.5 \mathrm{~kJ} \mathrm{~mol}^{-1}$ when adding salt in a wide range from 20 to $150 \mathrm{mM}$ concentration. This small effect due to ionic screening demonstrates that the net electrostatic contribution to binding is rather weak when compared, for instance, to the interaction of a polyelectrolyte (PE) chain to $\mathrm{HSA}^{33}$ Hence, we tentatively conclude that hydrophobic binding plays the dominant role.

A simple Debye-Hückel (DH) perspective for screened electrostatic interactions sketched in the following supports this conclusion. For this, consider the DH interaction between two spherical molecules $i$ and $j$ of valence $z_{i}$ and $z_{j}$ and radii $R_{i}$ and $R_{j}$, respectively, at a binding distance $r_{\text {bind }}=R_{\mathrm{t}}+R_{j}$, which reads $^{47-50}$

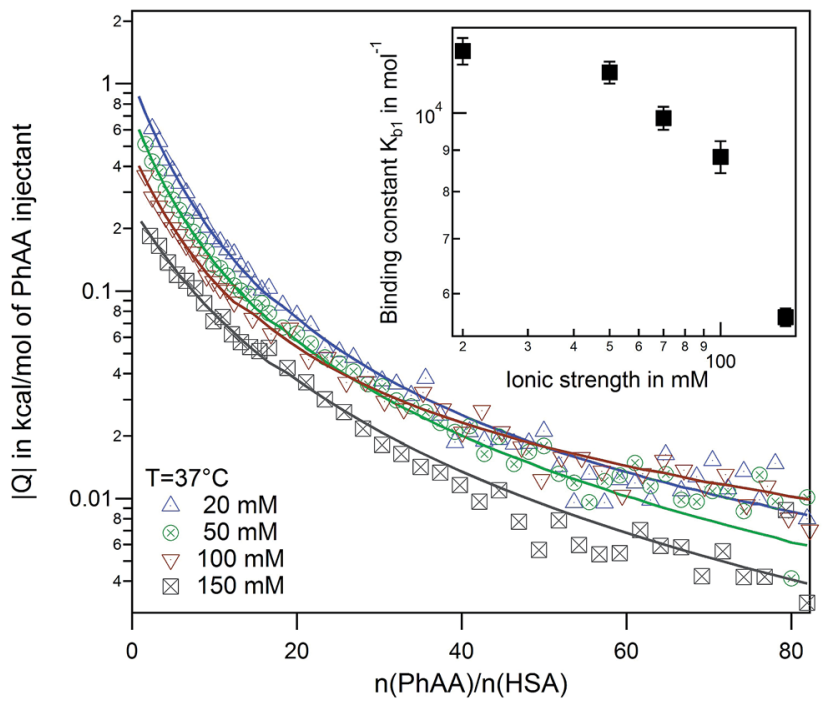

Fig. 5 Effect of ionic strength at $37^{\circ} \mathrm{C}$. Binding isotherms and corresponding fits for a series of ionic strengths from $I=20-150 \mathrm{mM}$ are shown. The inset displays the binding affinity in dependence of ionic strength.

$$
\Delta G_{\mathrm{DH}}^{i j} / k_{\mathrm{B}} T=\frac{z_{i} z_{j} \lambda_{\mathrm{B}}}{\left(R_{i}+R_{j}\right)\left(1+\kappa R_{i}\right)\left(1+\kappa R_{j}\right)} .
$$

Here, $\kappa=\sqrt{8 \pi \lambda_{B} c_{\text {salt }}}$ is the inverse Debye screening length and $\lambda_{\mathrm{B}} \approx 0.7 \mathrm{~nm}$ is the relevant Bjerrum length. We now assume two competing, leading order electrostatic contributions: first, the local attraction of the negatively charged ligand to the binding site at which a few positively charged amino acids are clustered together. Our previous work on the binding of a negative PE chain, for instance, suggested about 2-3 positive charges involved near the Sudlow II site. ${ }^{33}$ Second, the overall repulsion of the ligand to the sum of all the other charges on HSA which we assume to be located in the HSA center for simplicity. For the attractive term we take that the size of the charged groups at the binding site is on atomistic scales and the same as the ligand, i.e., $R_{\mathrm{PhAA}}=R_{\text {site }} \approx 0.2 \mathrm{~nm}$, such that the binding distance is $R_{\text {site }}+R_{\mathrm{PhAA}} \approx 0.4 \mathrm{~nm}$. The ligand valency is $z_{\mathrm{PhAA}}=-1$, and we assume $z_{\text {site }}=+2$. According to eqn (12) then the attractive term contributes approximately $-7 \mathrm{~kJ} \mathrm{~mol}^{-1}$ to the binding energy, decreasing in absolute magnitude with increasing ionic strength to about $-5.5 \mathrm{~kJ} \mathrm{~mol}^{-1}$. For the repulsive contribution, we model the protein as a charged sphere of radius $R_{\mathrm{HSA}} \approx$ $3.25 \mathrm{~nm}$ (ref. 51 and 52) and a net charge of $-14 \mathrm{e} . \S$ Thus, to compensate for the positive site, we obtain a remaining net valency of $z_{\mathrm{HSA}}=-16$ which we define to be located in the center of HSA. Due to the relatively large size of HSA, the electrostatic coupling ${ }^{53}$ is weak and within the validity of the DH treatment. The distance between the bound ligand and the protein center-of-charge is $R_{\mathrm{HSA}}=R_{\mathrm{PhAA}} \approx 3.45 \mathrm{~nm}$. According to eqn (12) this leads to a repulsive contribution of $\approx+3 \mathrm{~kJ}$ $\mathrm{mol}^{-1}$ at the lowest, and decreasing rapidly to only $\approx+1.5 \mathrm{~kJ}$

$\S$ The number of charges of the protein can be calculated from the crystal structure. 
$\mathrm{mol}^{-1}$ at the highest salt concentration. Hence, the net electrostatic interaction $\Delta G_{\mathrm{el}}=\Delta G_{\mathrm{DH}}^{\mathrm{HSA}-\mathrm{PhAA}}+\Delta G_{\mathrm{DH}}^{\text {site-PhAA }} \simeq-4 \pm 1$ $\mathrm{kJ} \mathrm{mol}^{-1}$ is attractive and small compared to the total experimental binding energies that are in the range between -25 and $-23 \mathrm{~kJ} \mathrm{~mol}^{-1}$ ( $c f$. Table 1, rounded), decreasing with increasing salt concentration.

Clearly, these numbers are highly approximative as, for instance, HSA is non-spherical and detailed charge asymmetry and excluded-volume effects on screening ${ }^{54}$ have been neglected. However, our estimates exemplify at least some order of magnitude and trends. The estimated attractive electrostatic contribution (between PhAA-site) to the binding is actually similar to monovalent ion pairing affinities found in explicit-water computer simulations. ${ }^{55}$ While the salt concentration dependence should be qualitatively correct, quantitative details are expected to be more complex than in our simple DH perspective due to very local hydration and polarization effects. ${ }^{55-57}$

We conclude that the electrostatic interaction is small and thus the major contribution must be of hydrophobic nature, stemming from interactions of the aromatic compound of PhAA (cf. Fig. 1) with the binding site. The total binding free can then be formally expressed as:

$$
\Delta G_{\mathrm{b}}=\Delta G_{\mathrm{phob}}+\Delta G_{\mathrm{el}}
$$

where $\Delta G_{\text {phob }}$ is the attractive hydrophobic contribution and $\Delta G_{\mathrm{el}}$ the net electrostatic interaction. Using the $\Delta G_{\mathrm{b} 1}$ values in Table 1 and subtracting the approximated values for $\Delta G_{\mathrm{el}}$ mentioned above, we obtain a hydrophobic contribution of the order of about $-19 \pm 1 \mathrm{~kJ} \mathrm{~mol}^{-1}$. The latter has comparable magnitude than the binding free energy calculated in explicitwater computer simulations of hydrophobic pocket-ligand binding at $298 \mathrm{~K}$ in pure water and with similar ligand size as in our study. ${ }^{58}$ It is also of comparable magnitude than the transfer free energy of a single benzene molecule from liquid water into its own liquid phase. ${ }^{59}$ Interestingly, the enthalpy calculated in the explicit-water simulations ${ }^{58}$ is $\Delta H=-29 \pm 17$ $\mathrm{kJ} \mathrm{mol}^{-1}$ and thus has the same sign and order of magnitude than $\Delta H_{\text {ITC }}=-20.3 \pm 0.6 \mathrm{~kJ} \mathrm{~mol}^{-1}$ measured in our experiments with $20 \mathrm{mM}$ salt (see ESI). This enthalpic signature is the reason for the inverse temperature dependency of $\Delta G_{\mathrm{b}}$ where the effect of hydrophobic attraction weakens with increasing temperature. Note that this is actually in contrast to what is typically known for hydrophobic association of simpler systems, which are entropy-driven ${ }^{60}$ and is argued to be due to the concave geometry of typical hydrophobic binding pockets. ${ }^{\mathbf{5 8 , 6 1 - 6 4}}$

\subsection{Binding of phenylacetic acid to modified HSA}

In the following we study the effect of carbamylation of HSA through urea ${ }^{35}$ on the binding affinity to PhAA under the same conditions as in the previous section. This in vitro modification of HSA is known to be found in patients suffering from chronic renal failure conditions, where the urea plasma concentration is chronically elevated. ${ }^{35}$ To study the urea-modification on the binding behavior of PhAA to HSA, we perform experiments again at $I=20 \mathrm{mM}$ and $I=150 \mathrm{mM}$ at the same three temperatures as before. Binding isotherms and corresponding

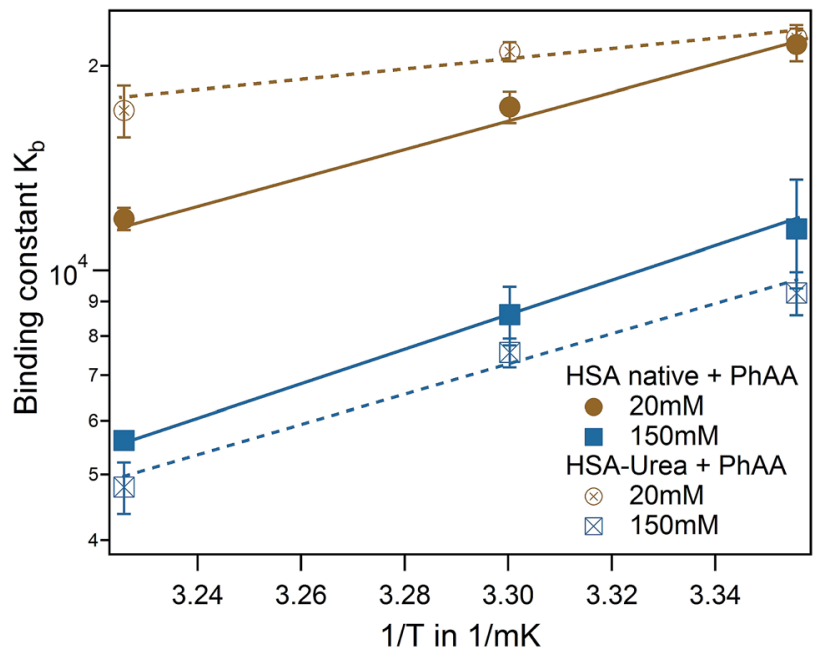

Fig. 6 Effect of HSA urea modification on PhAA adsorption. van't Hoff plot of the first binding process for native (filled symbols) and urea modified HSA (empty symbols) with corresponding fits.

fits are displayed in the ESI Fig. S2. $\dagger$ Results from the fits are summarized in Table S2 in the ESI $\uparrow$ and corresponding binding energies $\Delta G_{\mathrm{b}}$ using eqn (9) in Table 1.

For low ionic strength, binding to urea modified HSA apparently loses its temperature dependence while the strength of binding is principally unchanged. At high ionic strength and physiological condition, binding is even more weakened compared to $I=20 \mathrm{mM}$ for the modified system.

Binding isotherms are equally exothermic and exhibit similar curve shape as in Fig. 3 (data are shown in the ESI Fig. S2 $\dagger$ ). The binding affinity of the urea modified system to the native HSA system is compared for the first binding process and represented in a van't Hoff plot in Fig. 6.

For the binding of the second ligand at $20 \mathrm{mM}$ salt, no pronounced temperature dependence is observed in both of the systems (see Table 1). In both cases, binding of the second ligand is associated with a rather small affinity compared to the error of fitting. As a result, it is difficult to resolve a difference in binding affinities in the modified or native system or a corresponding temperature dependence.

\subsection{Binding of indoxyl sulfate to native and modified HSA}

The adsorption of IDS to native and modified HSA was systematically studied at two ionic strengths $I=20 \mathrm{mM}$ and $150 \mathrm{mM}$ and at three temperatures using ITC. Raw data and integrated heats for the adsorption of IDS to native HSA is depicted in Fig. S4 of the ESI. $\dagger$

Again all adsorption processes show similar exothermic isotherms as observed for PhAA adsorption in the section above. An increase in ionic strength from $20 \mathrm{mM}$ to $150 \mathrm{mM}$ show the same effect but overall weaker as observed before (Fig. S3b) in the ESI $\dagger$. However, in the standard ITC plot, temperature differences between different isotherms are not clearly visible (Fig. S4c) in the ESI $\dagger$. Therefore logarithmic plots of isotherms and corresponding fits are shown exemplarily for $I=150 \mathrm{mM}$ and three temperatures in Fig. 7a. 
Here the necessity to use a two site adsorption model is clear. As demonstrated in the case of IDS adsorption to native HSA at $37{ }^{\circ} \mathrm{C}$ and $I=150 \mathrm{mM}$ in Fig. $7 \mathrm{~b}, 9$ the data can neither be described by the SSIS (dotted line) nor the SBS (dashed line) model. There are clearly two binding sites present for IDS with different binding stoichiometry. Using the two set of independent sites TSIS model (solid line), ITC isotherms can be very well described at all measuring conditions. However, the number of IDS molecules adsorbed at the second site $\mathrm{N}_{2}$ cannot be well determined by a fit of the ITC data. Residual errors for fits with different fixed parameter $\mathrm{N}_{2}$ (ranging from one to three) show no significant change. It is however not surprising that fitting a monotonic curve with six parameters implies the risk of fitting of too many free parameters. ${ }^{65}$

Several studies on the adsorption of IDS to HSA confirm the existence of two binding sites with different binding affinity.,27 Crystallographic analysis of IDS complexed with HSA suggests that one IDS molecule bind to subdomain IIIA while two molecules bind to subdomain IIA of HSA. $^{66}$ Sakai et al. measured IDS adsorption on HSA at $25{ }^{\circ} \mathrm{C}$ and $67 \mathrm{mM}$ phosphate buffer at $\mathrm{pH} 7.4$ using equilibrium dialysis. They found two binding sites with $N_{1}=1$ and $N_{2}=3$ for high and low affinity binding site, respectively. ${ }^{3}$ A more recent study from Watanabe et al. using ultrafiltration found at same $\mathrm{pH}$ and temperature but different buffer, a slightly lower $N_{2}=1.6 .^{27}$ Based on these findings, it appears reasonable to fix $\mathrm{N}_{2}$ between one and three and average the results. As the residuals between each fits are small (see Fig. 7b), we use the standard deviation as error for each averaged parameter. Thus, even with an uncertainty of $N_{2}=2 \pm 1$, the binding affinity $k_{\mathrm{b} 2}$ obtained are robust and reliable. However, the binding enthalpy $\Delta H_{2}$ is strongly correlated with the fitting parameter $\mathrm{N}_{2}$ and errors for $\Delta H_{2}^{\mathrm{ITC}}$ and consequently $\Delta S_{2}$ are accordingly large. All results obtained from the fits are summarized in Table S3 of the ESI. $\dagger$

Binding of IDS to HSA is generally an order of magnitude stronger than PhAA to HSA in all measured cases. However, caution is required when comparing a macroscopic binding affinity $K_{\mathrm{b}}$ to a microscopic binding affinity $k_{\mathrm{b}}$. The microscopic affinity $k_{1}$ for example describes explicitly the binding process of $\mathrm{N}_{i}$ ligands to site 1 while the macroscopic affinity $K_{\mathrm{b} 1}$ assumes binding of either one of the two potential ligands to any of the two sites.

The effect of urea modification is rather small. van't Hoff analysis comparing native to urea modified HSA reveal temperature dependence of binding for both binding sites, while the dependency is more pronounced for the high affinity site rather than the low affinity site (Fig. 8). The binding enthalpies for the high affinity site measured directly by ITC $\Delta H_{1}^{\mathrm{ITC}}$ agree well with the binding enthalpies derived from the van't Hoff plot (see Table S3 of the ESI†).

A plot of all results comparing the effect of urea modification on the adsorption of PhAA and IDS is shown in Fig. 9. Additionally, the presented results are put into context to earlier investigations on the interaction between a multiply charged

I A further example of fitting quality is shown in the ESI (Fig. S5d $\dagger$ ) for modified HSA interaction to IDS.
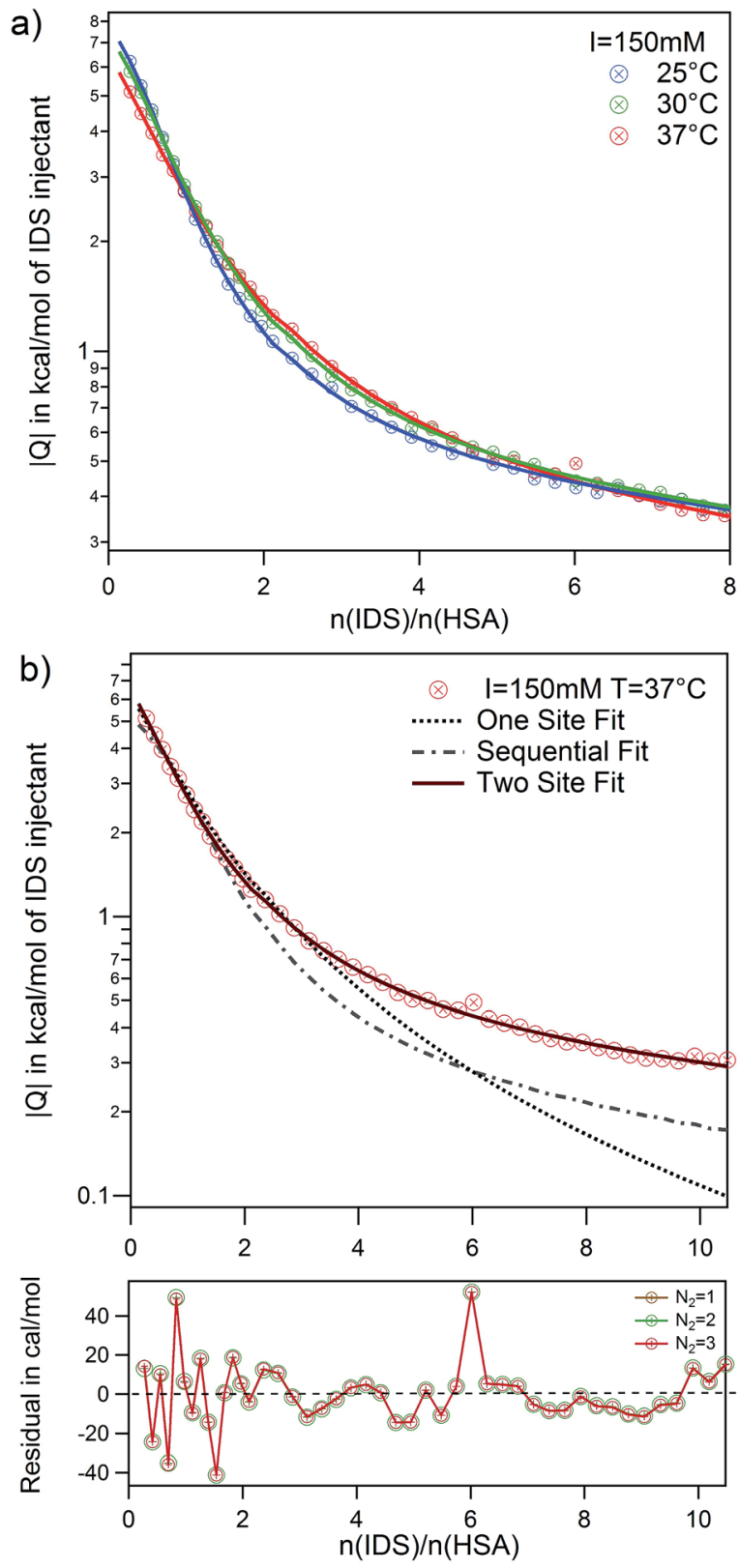

Fig. 7 Adsorption of IDS to native HSA at I = $150 \mathrm{mM}$. (a) Temperature series and corresponding fits using the TSIS model are shown. (b) Quality of different fit models and parameters are demonstrated by comparing three models SSIS (dotted line), SBS (dashed line) and TSIS (solid line) for $I=150 \mathrm{mM}$ and $37^{\circ} \mathrm{C}$. Beneath the graph is depicted the residual errors for TSIS fits with different fixed $\mathrm{N}_{2}$ values.

"middle molecule", namely a polyvalent polyacrylic acid (PAA) chain with HSA, to gain a deeper insight into different contributions to binding. ${ }^{33}$ In Fig. 9 the binding affinity of the small toxins IDS and PhAA to HSA exhibit in all cases moderate decreasing binding affinities to both native and modified HSA while binding of PAA is strongly weakened due to a decreasing counterion-release entropy with rising salt concentration. ${ }^{33}$ For the present ligands, the hydrophobic interaction plays an dominant role in the binding process as expected. 


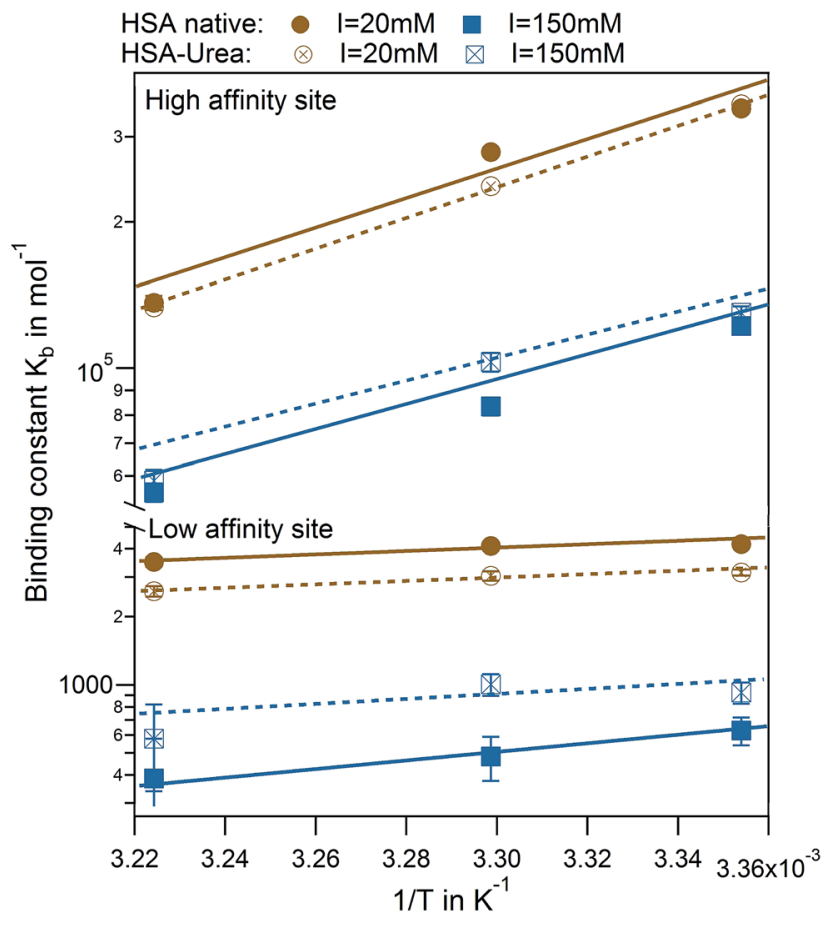

Fig. 8 van't Hoff plots for IDS interaction to HSA. High and low affinity binding sites comparing native (full symbols) and modified (empty symbols) HSA are shown for $I=20 \mathrm{mM}$ (brown) and $150 \mathrm{mM}$ (blue) with corresponding fits.

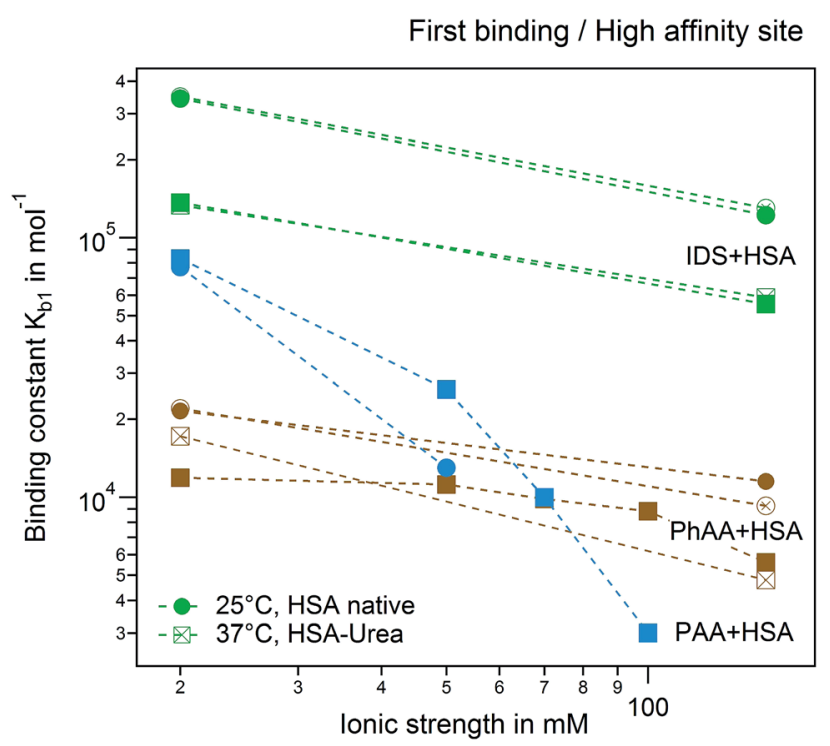

Fig. 9 lonic strength dependence of the first binding process and high affinity site. Adsorption of PhAA (brown), IDS (green) and PAA (blue) native (full symbols) and modified HSA (empty symbols) are shown. Spheres and rectangles represent $25^{\circ} \mathrm{C}$ and $37^{\circ} \mathrm{C}$.

\section{Conclusions}

We presented a study of the binding of two representative protein-bound uremic toxins indoxyl sulfate (IDS) and phenylacetic acid (PhAA) to native and urea modified HSA using ITC.
Binding of these ligands to HSA were in all cases exothermic and thus enthalpy driven. Analyzing the isotherms with appropriate binding models reveal that there are two binding sites for both of the two uremic toxins with different binding affinities and stoichiometry. Binding of PhAA to native HSA feature a subtle ionic strength dependency which can be estimated approximately by a Debye-Hückel approach. ${ }^{47}$ The main contribution towards binding is attributed to favorable hydrophobic interaction that can be well compared to computer simulations for a ligand-protein model system. ${ }^{58}$ The influence of urea modification of HSA towards binding appears to be rather weak and does not in principal alter interaction between ligand and protein.

\section{Acknowledgements}

The Helmholtz Virtual Institute for Multifunctional Biomaterials for Medicine are gratefully acknowledged for financial support. The authors thank the Helmholtz Association for funding of this work through Helmholtz-Portfolio Topic "Technology and Medicine". The authors thank B. Egbers for excellent technical assistance. J. D. thanks the Deutsche Forschungsgemeinschaft (DFG) for financial support. The authors acknowledge useful discussions with R. Gregor Wei $\beta$.

\section{References}

1 R. Vanholder, R. de Smet, G. Glorieux and W. Zidek, Kidney Int., 2003, 63, 1934-1943.

2 J. Jankowski, M. van der Giet, V. Jankowski, S. Schmidt, M. Hemeier, B. Mahn, G. Giebing, M. Tölle, H. Luftmann, H. Schlüter, W. Zidek and M. Tepel, J. Clin. Invest., 2003, 112, 256-264.

3 T. Sakai, K. Yamasaki, T. Sako, U. Kragh-Hansen, a. Suenaga and M. Otagiri, Pharm. Res., 2001, 18, 520-524.

4 L. Viaene, P. Annaert, H. de Loor, R. Poesen, P. Evenepoel and B. Meijers, Biopharm. Drug Dispos., 2013, 34, 165-175.

5 T. Sakai, A. Takadate and M. Otagiri, Biol. Pharm. Bull., 1998, 18, 1755-1761.

6 Y. Itoh, A. Ezawa, K. Kikuchi, Y. Tsuruta and T. Niwa, Anal. Bioanal. Chem., 2012, 403, 1841-1850.

7 R. Vanholder, N. Meert, E. Schepers, G. Glorieux, A. Argiles, P. Brunet, G. Cohen, T. Drüeke, H. Mischak, G. Spasovski, Z. Massy and J. Jankowski, Nephrol., Dial., Transplant., 2007, 22, 3115-3121.

8 O. Deltombe, W. Van Biesen, G. Glorieux, Z. Massy, A. Dhondt and S. Eloot, Toxins, 2015, 7, 3933-3946.

9 P. Gulyassy and T. Depner, Am. J. Kidney Dis., 1983, 2, 578601.

10 X. Li and Y. Yan, J. Mol. Struct., 2015, 1082, 170-173.

11 M. Ishtikhar, G. Rabbani and R. H. Khan, Colloids Surf., B, 2014, 123, 469-477.

12 M. T. Rehman, H. Shamsi and A. U. Khan, Mol. Pharm., 2014, 11, 1785-1797.

13 N. Zaidi, M. R. Ajmal, G. Rabbani, E. Ahmad and R. H. Khan, PLoS One, 2013, 8, e71422. 
14 F. Brettschneider, M. Tölle, M. von der Giet, J. PasslickDeetjen, S. Steppan, M. Peter, V. Jankowski, A. Krause, S. Kühne, W. Zidek and J. Jankowski, Artif. Organs, 2013, 37, 409-416.

15 R. C. Vanholder, S. Eloot and G. L. Glorieux, Am. J. Kidney Dis., 2016, 67, 664-676.

16 F. Böhringer, V. Jankowski, P. R. Gajjala, W. Zidek and J. Jankowski, ASAIO J., 2015, 61, 55-60.

17 J. Jankowski, W. Zidek, V. Brettschneider and S. Jankowski, Method of dialysis for removing protein-bound toxins from the blood of patients using high-frequency electromagnetic fields, 2014.

18 S. Leavitt and E. Freire, Curr. Opin. Struct. Biol., 2001, 11, 560-566.

19 V. Ball and C. Maechling, Int. J. Mol. Sci., 2009, 10, 32833315.

20 J. E. Ladbury, Biochem. Soc. Trans., 2010, 38, 888-893.

21 L. S. Roselin, M.-S. Lin, P.-H. Lin, Y. Chang and W.-Y. Chen, Biotechnol. J., 2010, 5, 85-98.

$22 \mathrm{X}$. Li and Z. Yang, Chem.-Biol. Interact., 2015, 232, 77-84.

23 S. Tayyab, M. S. Zaroog, S. R. Feroz, S. B. Mohamad and S. N. A. Malek, Int. J. Pharm., 2015, 491, 352-358.

24 B. K. Paul, N. Ghosh and S. Mukherjee, J. Phys. Chem. B, 2015, 119, 13093-13102.

25 P. Sen, S. Fatima, B. Ahmad and R. H. Khan, Spectrochim. Acta, Part A, 2009, 74, 94-99.

26 L.-L. Wu, H.-W. Gao, N.-Y. Gao, F.-F. Chen and L. Chen, BMC Struct. Biol., 2009, 9, 31.

27 H. Watanabe, T. Noguchi, Y. Miyamoto, D. Kadowaki, S. Kotani, M. Nakajima, S. Miyamura, Y. Ishima, M. Otagiri and T. Maruyama, Drug Metab. Dispos., 2012, 40, 1423-1428.

28 C. Ràfols, S. Zarza and E. Bosch, Talanta, 2014, 130, 241-250.

29 H. Xu, X.-D. Yu, X.-D. Li and H.-Y. Chen, Chromatographia, 2005, 61, 419-422.

30 R. Tian, S. Xu, X. Lei, W. Jin, M. Ye and H. Zou, TRAC, 2005, 24, 810-825.

31 N. Bijari, Y. Shokoohinia, M. R. Ashrafi-Kooshk, S. Ranjbar, S. Parvaneh, M. Moieni-Arya and R. Khodarahmi, J. Lumin., 2013, 143, 328-336.

32 N. Keswani, S. Choudhary and N. Kishore, J. Biochem., 2010, 148, 71-84.

33 S. Yu, X. Xu, C. Yigit, M. van der Giet, W. Zidek, J. Jankowski, J. Dzubiella and M. Ballauff, Soft Matter, 2015, 11, 46304639.

34 M. Rossi, K. L. Campbell, D. W. Johnson, T. Stanton, D. A. Vesey, J. S. Coombes, K. S. Weston, C. M. Hawley, B. C. McWhinney, J. P. J. Ungerer and N. Isbel, Arch. Med. Res., 2014, 45, 309-317.

35 A. H. Berg, C. Drechsler, J. Wenger, R. Buccafusca, T. Hod, S. Kalim, W. Ramma, S. M. Parikh, H. Steen, D. J. Friedman, J. Danziger, C. Wanner, R. Thadhani and S. A. Karumanchi, Sci. Transl. Med., 2013, 5, 175 ra29.

36 L. MicroCal, ITC Data Analysis in Origin, $7^{\text {th }}$ edn, 2004.

37 J. Tellinghuisen and J. D. Chodera, Anal. Biochem., 2011, 414, 297-299.
38 S. G. Krimmer and G. Klebe, J. Comput.-Aided Mol. Des., 2015, 29, 867-883.

39 S. Geschwindner, J. Ulander and P. Johansson, J. Med. Chem., 2015, 58, 6321-6335.

40 L. Indyk and H. F. Fisher, Methods Enzymol., 1998, 295, 350364.

41 D. T. Haynie, Biological Thermodynamics, Cambridge University Press, 2001.

42 G. G. Hammes, Thermodynamics and kinetics for the biological sciences, Wiley-Interscience, John Wiley \& Sons. Inc., 2000.

43 S. Vega, O. Abian and A. Velazquez-Campoy, Methods, 2015, 76, 99-115.

44 I. Herrera and M. a. Winnik, J. Phys. Chem. B, 2013, 117, 8659-8672.

45 E. Ahmad, G. Rabbani, N. Zaidi, S. Singh, M. Rehan, M. M. Khan, S. K. Rahman, Z. Quadri, M. Shadab, M. T. Ashraf, N. Subbarao, R. Bhat and R. H. Khan, PLoS One, 2011, 6.

46 T. Chatterjee, A. Pal, S. Dey, B. K. Chatterjee and P. Chakrabarti, PLoS One, 2012, 7, e37468.

47 P. Debye and E. Hückel, Phys. Z., 1923, 24, 185-206.

48 M. B. Jackson, Molecular and Cellular Biophysics, Cambridge University Press, 2006.

49 G. G. Hammes, Thermodynamics and kinetics for the biological sciences, Wiley-Interscience, John Wiley \& Sons. Inc., 2000.

50 C. Yigit, J. Heyda, M. Ballauff and J. Dzubiella, J. Chem. Phys., 2015, 143, 064905.

51 A. Luik, Y. Naboka, S. Mogilevich, T. Hushcha and N. Mischenko, Spectrochim. Acta, Part A, 1998, 54, 1503-1507.

52 M. A. Kiselev, I. A. Gryzunov, G. E. Dobretsov and M. N. Komarova, Biofizika, 2001, 46, 423-427.

53 H. Boroudjerdi, Y.-W. Kim, A. Naji, R. Netz, X. Schlagberger and A. Serr, Phys. Rep., 2015, 416, 129.

54 N. Hoffmann, C. N. Likos and J.-P. Hansen, Mol. Phys., 2004, 102, 857-867.

55 I. Kalcher and J. Dzubiella, J. Chem. Phys., 2009, 130, 134507.

56 J. Dzubiella, J. Am. Chem. Soc., 2008, 130, 14000.

57 J. M. Scholtz, H. Qian, V. H. Robbins and R. L. Baldwin, Biochemistry, 1993, 32, 9668-9676.

58 P. Setny, R. Baron and J. A. Mccammon, J. Chem. Theory Comput., 2010, 6, 2866-2871.

59 R. S. Spolar, J.-H. Ha and T. Record Jr, Proc. Natl. Acad. Sci. U. S. A., 1989, 86, 8382 .

60 C. Tanford, The hydrophobic effect: formation of micelles and biological membranes, New York, Wiley, 1973.

61 G. Hummer, Nat. Chem., 2010, 2, 906.

62 J. Dzubiella, J. Stat. Phys., 2011, 145, 227.

63 F. Biedermann, W. M. Nau and H.-J. Schneider, Angew. Chem., Int. Ed., 2014, 53, 11158-11171.

64 B. Breiten, M. R. Lockett, W. Sherman, S. Fujita, M. Al-Sayah, H. Lange, C. M. Bowers, A. Heroux, G. Krilov and G. M. Whitesides, J. Am. Chem. Soc., 2013, 135, 15579-15584. 65 C. A. Brautigam, Methods, 2015, 76, 124-136.

66 J. Ghuman, P. A. Zunszain, I. Petitpas, A. A. Bhattacharya, M. Otagiri and S. Curry, J. Mol. Biol., 2005, 353, 38-52. 